\section{Developmental expression of inositol 1, 4, 5-trisphosphate receptor in the post-natal rat cochlea}

\author{
W. J. Liu, J. Yang \\ Department of Otorhinolaryngology- \\ Head \& Neck Surgery, Xinhua Hospital, \\ Shanghai Jiaotong University School \\ of Medicine, Shanghai Jiaotong \\ University School of Medicine Ear \\ Institute, Shanghai Key Laboratory of \\ Translational Medicine on Ear and Nose \\ diseases, Shanghai, China
}

\section{Abstract}

Inositol 1, 4, 5-trisphosphate receptor (IP3R) has been established to be essential for hearing. However, the expression of IP3R in the cochlea in the period of auditory development remains unknown. We investigated the expression of IP3R in the developing rat cochlea using immunohistochemistry and real-time reverse transcription polymerase chain reaction (RT-PCR). We observed its presence in the developing rat cochlea, and changes in IP3R protein expressions from the early post-natal period to adult. At birth (postnatal day $0, P 0)$, IP3R expression was only found in Hensen's cell. IP3R immunoreactivity first appeared in the sensory hair cells in the organ of Corti at P2. This localization was confirmed by means of double-labeling experiments with Myosin VIIA, a marker for cochlear hair cells. Colocalization of IP3R and Myosin VIIA from P2 to the second post-natal week suggested early expression of IP3R in developing inner and outer hair cells. Claudius' cells near the spiral ligament were labelled for IP3R from P8 onwards. Transient IP3R expression was observed in the stria vascularis in early post-natal rat from P4 to P8. Spiral ganglion neurons also exhibited weaker IP3R fluorescence signals during post-natal development. The results of RT-PCR demonstrated that all three IP3R isoforms (IP3R1, IP3R2, and IP3R3) were present in rat cochlea during four different developmental stages of cochlea, from P0 to P28. Present immunohistochemical evidence for both change and maintenance of expression of IP3R during post-natal development of the rat cochlea indicated the possible involvement of IP3R-mediated calcium signaling in cochlear development.

\section{Introduction}

According to the theory of P2 purinoceptor / phospholipase C (PLC) / inositol 1, 4, 5trisphosphate (IP3) / $\mathrm{Ca}^{2+}$ signaling cascade, supporting cells within Kölliker's organ in the immature cochlea release ATP through the gap junctional hemichannel, ATP excites $\mathrm{G}$ protein coupled P2 purinoceptor on adjacent cells that produce PLC dependent IP3, $\mathrm{Ca}^{2+}$ releases from the intracellular calcium stores induced by the IP3 receptor (IP3R), then $\mathrm{Ca}^{2+}$ signal propagates among cells. ${ }^{1,2}$ Numerous and diverse aspects of cochlear physiology are controlled by calcium signaling that derives mainly from two pathways, namely, $\mathrm{Ca}^{2+}$ release from intracellular stores and $\mathrm{Ca}^{2+}$ influx through ion channels. The former is ascribed to both the IP3R and the ryanodine receptor (RyR). ${ }^{3}$ The role of $\mathrm{Ca}^{2+}$ released from intracellular stores in hearing was confirmed in a mutant connexin 26 (Cx26) model in which the impaired intercellular transfer of IP3 caused recessive hereditary deafness. ${ }^{4}$ IP3 is a $\mathrm{Ca}^{2+}$-mobilizing second messenger, and signals by binding to the IP3R. ${ }^{5}$ Three mammalian subtypes of IP3R (IP3R type 1,2, and 3), deriving from three different genes, have been identified by molecular cloning. ${ }^{6}$ They have the same IP3 binding site, showing the same $\mathrm{Ca}^{2+}$ gating, and ionic conductance. $^{7}$

Despite the indispensable role of IP3R in the signaling cascade, the expression of IP3R is undetermined in the developing cochlea, and not systematically reported in the mature cochlea, according to previous reports. For example, using immunofluorescence staining, Gossman et al. ${ }^{8}$ reported that IP3Rs were extensively expressed in the cochlear sensory epithelium in which intense labeling for IP3Rs was visible in the cytoplasm and on the cochlear cell surface in adult guinea pig. However, the precise localization of IP3R was not available in their results. In another study, IP3R was localized to the lateral wall of the outer hair cells $(\mathrm{OHCs})$ in whole-mount preparations of the organ of Corti also in adult guinea pig through immunofluorescence staining. A patch clamp study by Sugasawa et $a l .{ }^{9}$ showed that intracellular heparin, an inhibitor of IP3 receptors, significantly reduced the ATP-evoked $\mathrm{Ca}^{2+}$ response in inner hair cells (IHCs), which suggested that IP3R is expressed in IHCs. Obviously, the knowledge of the spatiotemporal expression of IP3R in the developing and mature cochlea would provide the basis for further understanding how IP3R-mediated calcium signaling contributes to cochlear function. In addition, IP3R expression has been shown to be associated with proliferation, differentiation, and development in many cell types. ${ }^{10-13}$
Correspondence: Dr. Jun Yang, Department of Otorhinolaryngology-Head \& Neck Surgery, Xinhua Hospital, Shanghai Jiaotong University School of Medicine, Shanghai Jiaotong University School of Medicine Ear Institute, Shanghai Key Laboratory of Translational Medicine on Ear and Nose diseases, Shanghai 200092, China.

Tel. +86.21.25078532 - Fax: +86.21.65156489.

E-mail: otology-xinhua@hotmail.com

Key words: Inositol 1, 4, 5-trisphosphate receptor, immunohistochemistry, development, cochlea, rat.

Contributions: WL, laboratory experiments, results analysis and interpretation, manuscript writing; JY, research theme definition, methods design, manuscript revision. All authors approved the version to be published.

Funding: this study was supported by the National Natural Science Foundation of China (No. 81170919, No. 81470689), the project of Shanghai Municipal Science and Technology Commission (No. 14DJ1400201, No. 14DZ2260300).

Conflict of interest: the authors declare no potential conflict of interest.

Received for publication: 19 January 2015.

Accepted for publication: 4 April 2015.

This work is licensed under a Creative Commons Attribution NonCommercial 3.0 License (CC BYNC 3.0).

(C) Copyright W. J. Liu and J. Yang, 2015

Licensee PAGEPress, Italy

European Journal of Histochemistry 2015; 59:2486 doi:10.4081/ejh.2015.2486

It would be of interest to know whether the expression of IP3R was related to cochlear development. In our previous study, we determined the distribution of RYR in developing rat cochlea and provided direct evidence implicating RYR as an important regulator of cochlear maturity. ${ }^{14}$ Therefore, we carried out a detailed analysis of the expression pattern of IP3R in the developing and adult rat cochlea using immunofluorescence in the present study. Since the antibody used for IP3R characterization does not discriminate each isoform of IP3R, the expression of three IP3R isoforms in the rat cochlea during post-natal development was studied by RT-PCR.

\section{Materials and Methods}

\section{Animals}

Male and female Sprague-Dawley rats of the following ages were examined for this study: 
post-natal day 0 (P0), P2, P4, P8, P14, P17, P21, P28 and the adult. The experimental procedures of the present study were performed in accordance with the regulations of the Animal Use and Care Committee of Shanghai Jiaotong University School of Medicine. Post-natal rats were anesthetized by an intraperitoneal injection of $10 \%$ chloral hydrate $(0.2 \mathrm{~mL} / 100 \mathrm{~g})$.

\section{Western blotting}

Western blotting was performed using extracts from samples of the entire adult cochlea. A whole brain was removed from one animal. Tissues obtained from the two different regions were homogenized separately in ice-cold RIPA Lysis Buffer (50 mM Tri-HCl, pHs 7.6, $150 \mathrm{mM} \mathrm{NaCl}, 0.1 \% \mathrm{SDS}, 1 \% \mathrm{NP} 40$, and $0.1 \mathrm{mM}$ EGTA). Homogenates were fractionated by SDS-PAGE and electrophoretically transferred onto a nitrocellulose membrane. Membranes were blocked with 5\% w/v nonfat dry milk and immunoblotting was performed using rabbit anti-IP3 polyclonal antibodies (1:1000; Abcam, Cambridge, MA, USA) and anti- $\beta$-Actin monoclonal antibody (1:1000; Biyuntian, Hangzhou, China) served as an internal loading control. The protein bands were visualized by the application of horseradish peroxidase-conjugated secondary antibody, and were detected using a chemiluminescent reagent (Biyuntian). Reaction product levels

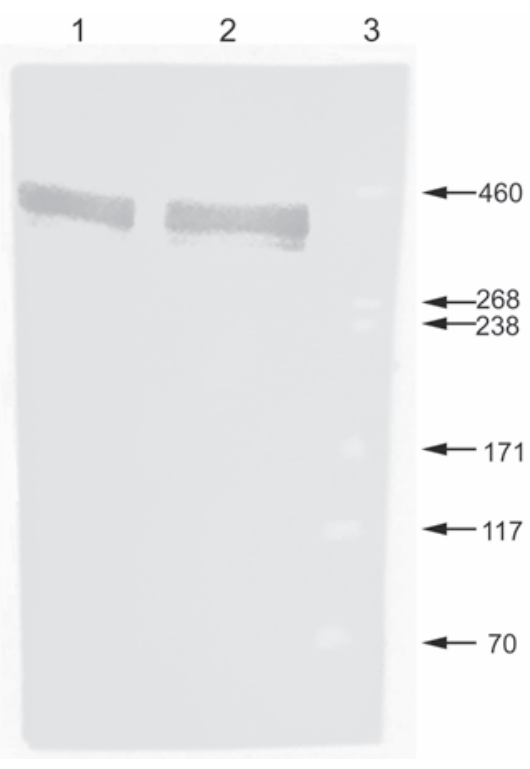

Figure 1. Western blotting assessment of the specificity of the anti-IP3R polyclonal antibody. Lane 1 indicates cochlear tissue samples from adult rat, showing a strong band of about $314 \mathrm{kD}$ molecular weight. Lane 2 represents the positive control rat brain, showing also a single band at size of approximate $314 \mathrm{KD}$. Lane 3 shows the molecular weight standards. were quantified by the Quantity One System (Bio-Rad, Hercules, CA, USA).

\section{Immunohistochemistry}

Since the reliability of our fixative protocol was confirmed in a previous study, ${ }_{15}$ the present study continued to follow it. Post-natal rats at different developmental stages (P0, P2, P4, P8, P14, P17, P21, and adult) were fixed initially by intracardiac perfusion with ice-cold $4 \%$ paraformaldehyde in $0.1 \mathrm{M}$ phosphate buffer $(\mathrm{pH}$ 7.4) and the cochlea was then quickly removed. The round and oval windows of the cochlea were opened, and a small hole made in the bony apex of the cochlea. After perilymphatic perfusion with the above fixative, cochleae were post-fixed in the same fixative for $35 \mathrm{~min}$ at room temperature. P5 and older animals were decalcified in $10 \%$ EDTA at pH 7.4. The cochleae ware thoroughly rinsed with $0.01 \mathrm{M} \mathrm{PBS}$ and subsequently placed in a gradient of sucrose $15 \%$ for $3 \mathrm{~h}$ and $30 \%$ overnight. The cochleae were then embed-
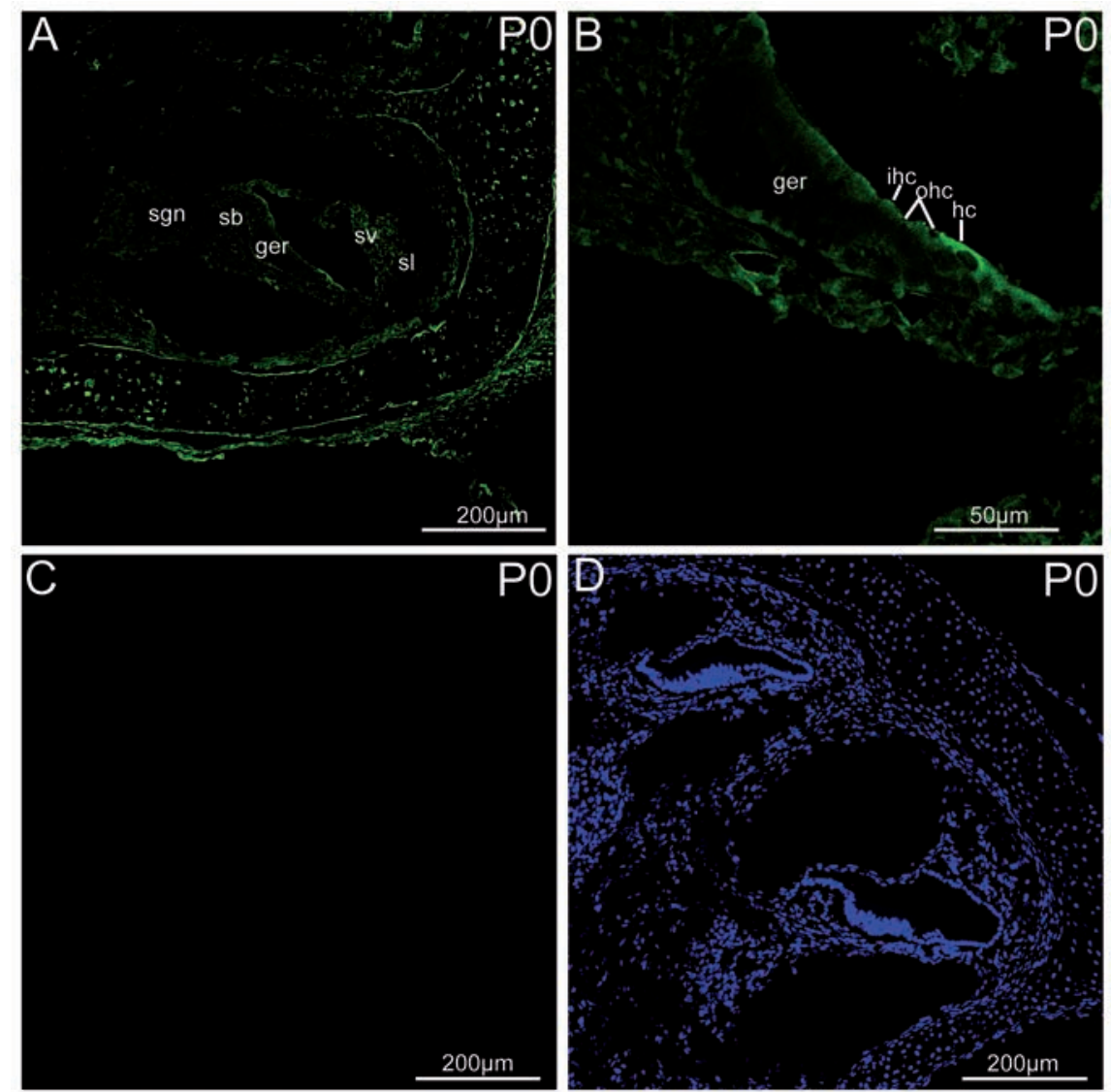

ded in optimum cutting temperature compound (OCT) at $4^{\circ} \mathrm{C}$ for $3 \mathrm{~h}$. Serial 6 - $\mu \mathrm{m}$-thick sections were cut with a cryostat microtome. The frozen sections were pre-incubated in $10 \%$ normal donkey serum, $0.3 \%$ TritonX-100 in PBS for $30 \mathrm{~min}$ at room temperature, followed by incubation with the polyclonal IP3 receptor antibodies (1:250; Abcam) in 5\% normal donkey serum and $0.1 \%$ Triton X-100 in 0.01 M PBS overnight at $4^{\circ} \mathrm{C}$. After rinse in $0.01 \mathrm{M} \mathrm{PBS}$, the sections were incubated in Alexa 488-conjugated donkey antirabbit antibodies (1:200; Jackson ImmunoResearch, West Grove, PA, USA) at $37^{\circ} \mathrm{C}$ for $1 \mathrm{~h}$. For IP3R and myosin VIIA double labeling of cryosections of the cochlea from $\mathrm{P} 0$ to $\mathrm{P} 14$ rat, anti-IP3R antibody was mixed with mouse antimyosin VIIA antibody (1:200; Hybridoma Bank, Iowa City, IA, USA), in PBS containing $0.1 \%$ Triton X-100 and 5\% normal donkey serum, overnight at $4^{\circ} \mathrm{C}$. Negative control sections were processed with PBS instead of the primary antibodies. After washing in PBS, the sections were

\footnotetext{
Figure 2. Confocal images of IP3R immunolabeling in the mid turn of the rat cochlea at P0. A) An overview of IP3R immunolabeling in the mid turn of the rat cochlea at P0; IP3R immunolabeling was only present in the Hensen's cells; no IP3R immunoreactivity was detected in other types of cochlear cells. B) Detail of P0 organ of Corti showed IP3R immunolabelling in the Hensen's cells that are adjacent to the outermost row of the outer hair cells. C,D) The negative control using anti-rabbit IgG antibody in a single labeling experiment showed no staining. ger, greater epithelial ridge; ihc, inner hair cells; ohc, outer hair cells; hc, Hensen's cells; sgn, spiral ganglion neuron; sl, spiral ligament; sv, stria vascularis.
} 
incubated with a mixture of Alexa 488-conjugated donkey anti-rabbit (1:200; Jackson) and Alexa 594-conjugated donkey anti-mouse antibodies (1:200; Jackson) secondary antibodies. Sections were rinsed, nuclei were visualized with 4', 6diamidino-2-phenylindole (DAPI). Images were photographed on a laser confocal scanning microscopes (LSM 710; Zeiss, Jena, Germany) using Zeiss ZEN 2010 software, images were processed using Adobe Photoshop software.

\section{RNA isolation and quantitative RT-PCR}

Rats at four different developmental stages (P0, P8, P14, and P28) were decapitated, and the cochlea was collected from 5 animals at each developmental period. Total RNA extraction was performed using Trizol Reagent (Invitrogen, Carlsbad, CA, USA), according to the manufacturer's instructions. Reverse transcription was carried out using the RevertAid First Strand cDNA Synthesis Kit (Fermentas, Hanover, MD, USA). PCR amplification was carried out using the following primers:

IP3R1, 5 -CCTCTTCCGGTTCAAGCACCTGG3 (forward) and 5 -GCATTTCTCAACCTGCTCCGAGATG -3 (reverse);

IP3R2, 5 - CCGTACAAGTACGTGCTGCGTC TC-3 (forward) and 5 - GCCGATCTGAGACTGCATGACGC-3 (reverse);

IP3R3, 5 - CGTGGTCGGCGAGGCCGTG-3 (forward) and 5 - CGCAGCAGCTCGCTGAAGCTG -3 (reverse); and $\beta$-actin, 5 -GACCTTCAATGTCCCTGCCATG-3 (forward) and 5 GATAGGCACGTTGTGAGTCACGC-3 (reverse) (from Invitrogen).

The amplification parameters were as follows: $95^{\circ} \mathrm{C}$ pre-denaturation ( $1 \mathrm{~min}$ ), followed by 40 cycles at $95^{\circ} \mathrm{C}(15 \mathrm{~s}), 58^{\circ} \mathrm{C}(20 \mathrm{~s})$, and $72^{\circ} \mathrm{C}(20 \mathrm{~s})$, with a final extension at $72^{\circ} \mathrm{C}(5$ min), and a melting curve analysis $\left(72-95^{\circ} \mathrm{C}\right.$, increments of $1^{\circ} \mathrm{C}$ ) to confirm primer specificity. Data were analyzed using the $\Delta \Delta \mathrm{C}_{\mathrm{T}}$ method, and the $\beta$-actin gene was used as an endogenous internal control at all stages. The threshold cycle $\left(\mathrm{C}_{\mathrm{T}}\right)$ was normalized to the housekeeping $\beta$-actin gene, and the $2^{-\Delta \Delta C T}$ method was used to calculate changes in gene expression.

\section{Statistical analyses}

Comparison of mRNA levels of IP3R1, IP3R2, and IP3R3 at the various developmental stages was made using analysis of variance. Comparison of mRNA levels among IP3R1, IP3R2, and IP3R3 at the corresponding developmental stages was made using analysis of variance as well. The Brown-Forsythe version of analysis of variance $\left[F\left({ }_{B F}\right)\right]$ was used, while the assumption of homogeneity of variance was rejected.

\section{Results \\ Antibody specificity}

Western blotting analysis was performed to confirm the specificity of the anti-IP3R antibody in the present study. A band with about $314 \mathrm{kDa}$ molecular weight was recognized by the polyclonal anti-IP3R antibody in both the cochlea and the brain (as a control tissue) in adult rat (Figure 1). These findings demonstrated that this antibody was suitable for immunohistochemistry.

\section{Localization of IP3R in the rat cochlea during development by confocal microscopy}

The immunolocalization of IP3R was inves-
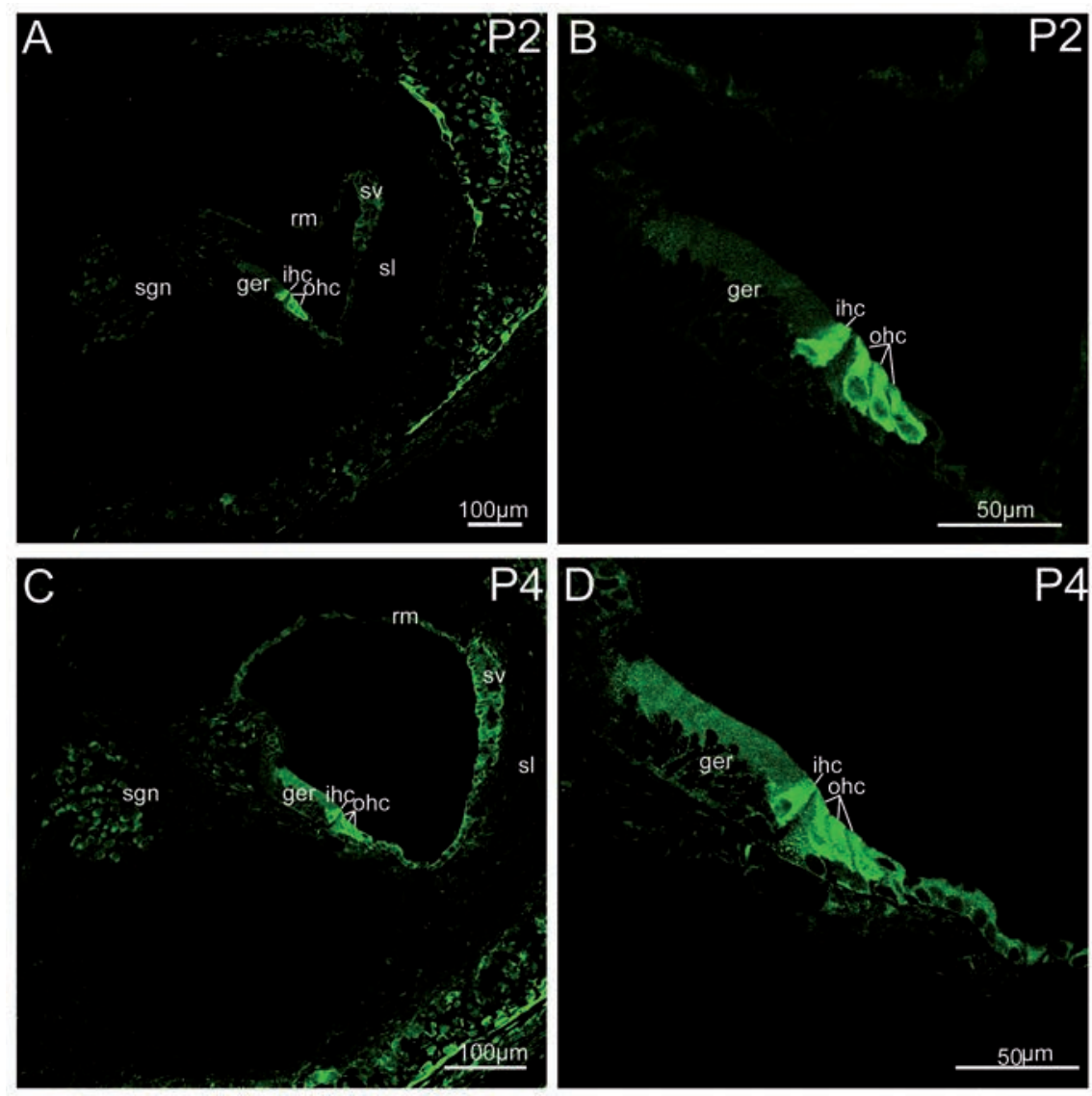

Figure 3. Confocal images of IP3R immunolabeling in the mid turn of the rat cochlea at P2 and P4. A) An overview of IP3R immunolabeling in the mid turn of the rat cochlea at P2; note that IP3R immunoreactivity started to appear in the inner and outer hair cells; virtually no IP3R immunoreactivity was detected in other types of cochlear cells. B) Detail of IP3R expression in the P2 organ of Corti; pronounced IP3R immunoreactivity was detected in the bodies of inner hair cells and outer hair cells; note that IP3R immunoreactivity disappeared from the Hensen's cells. C) An overview of IP3R immunolabeling in the mid turn of the rat cochlea at P4; intense IP3R immunostaining was observed in the inner and outer hair cells; in addition, IP3R immunostaining occurred in the stria vascularis and spiral ganglion neurons. D) Detail of IP3R expression in the P4 organ of Corti; IP3R immunolabeling was found in both types of hair cells. ger, greater epithelial ridge; ihc, inner hair cells; ohc, outer hair cells; rm, Reissner's membrane; sgn, spiral ganglion neuron; sl, spiral ligament; sv, stria vascularis. 
lar membranes, the spiral vessel underneath the basilar membrane, spiral ligament, and spiral limbus (not shown). Previously, it has been proposed that mouse IgG, but not IgM, can produce artifactual diffuse staining in the spiral limbus, the basilar membrane, and beneath the external sulcus cells. ${ }^{16}$

Until P2, no IP3R immunoreactivity was detected in both IHCs and OHCs in the organ of Corti, whereas IP3R immunolabeling disappeared from Hensen's cell (Figure 3 A,B). At P4, IP3R immunostaining was similar to that described for P2, the most notable immunolabeling was observed in the IHCs and OHCs. Apart from this, immunolabelling for IP3R was observed in the spiral ganglion neurons (SGNs) and stria vascular (Figure 3 C,D). At $\mathrm{P} 8$, strong immunolabelling was still present in the sensory hair cell regions, however, the SGNs and the stria vascular continued to express IP3R though weak. Even more impressively, IP3R immunostaining extended laterally to include the Claudius' cells (Figure 4 A,B). At the onset of hearing (P14), little or no labeling appeared in the SGNs and the stria vascular. It appeared that IP3R expression was constrained in the organ of Corti. Uniform distribution of IP3R immunolabeling was observed in the cytoplasm of the IHCs, from the base to the apex. However, immunolabeling in the OHCs was found in the cytoplasmic region between the cuticular plate and the nucleus. A lesser degree of IP3R expression was observed in the Deiters' and Claudius' cells (Figure 4 C,D).

To further clarified whether IP3R is specifically expressed in early post-natal cochlear hair cells, cryosections of the rat cochlea from P0 to P14 rat were co-labeled with antibodies against IP3R and myosin VIIA, the latter proven to be a reliable marker for developing hair cells. ${ }^{17-19}$ The results revealed that substantial colocalization of IP3R and myosin VIIA in P2-P14 cochlear hair cells, while no coexpression of IP3R and myosin VIIA could be detected in cochlear hair cells in the $\mathrm{P} 0$ rat (Figure 5 A-0).

Beyond the second post-natal week to the adult, IP3R expression profile exhibited only minor changes. At P17, IP3R expression remained restricted to the organ of Corti. IP3R immunolabeling was observed in the IHCs and OHCs, the basal region of the Deiters' cells adjacent to basilar membrane and the Claudius' cells (Figure 6 A,B). At later postnatal stage (at P21), sensory hair cells staining was still pronounced in the cytoplasmic. In the Deiters' cells, the cytoplasmic region beneath the nucleus was immunolabelled (Figure $6 \mathrm{C}, \mathrm{D}$ ). In the P17-P21 period, the expression of IP3R was still much weaker in SGNs than hair cells (Figure $6 \mathrm{E}, \mathrm{F}$ ). In adult, a more homogenous IP3R immunolabeling was distributed throughout the cytoplasm of IHCs and OHCs, including both basal and apical regions. An increased immunolabeling was observed in Deiters' cells. In addition, inner sulcus cells and Claudius' cells exhibited moderate immunostaining (Figure 7 A,B). Omission of primary antisera resulted in loss of these specific labeling profiles in cochlear tissues in adult (Figure 7 C,D).

\section{Expression of mRNAs for IP3R1, IP3R2, and IP3R3 in the rat cochlea}

The results shown in Figure 8 demonstrated that mRNAs for all the three isoforms of IP3Rs were present in the whole rat cochlea. There was no difference in expressions of IP3R1, IP3R2, and IP3R3 mRNA at the various developmental stages (one-way ANOVA, $\mathrm{F}_{\mathrm{IP} 3 \mathrm{R} I}=0.679, \mathrm{P}=0.589 ; \mathrm{F}_{\mathrm{IP} 3 \mathrm{R} 2}=0.241, \mathrm{P}=0.865 ;$
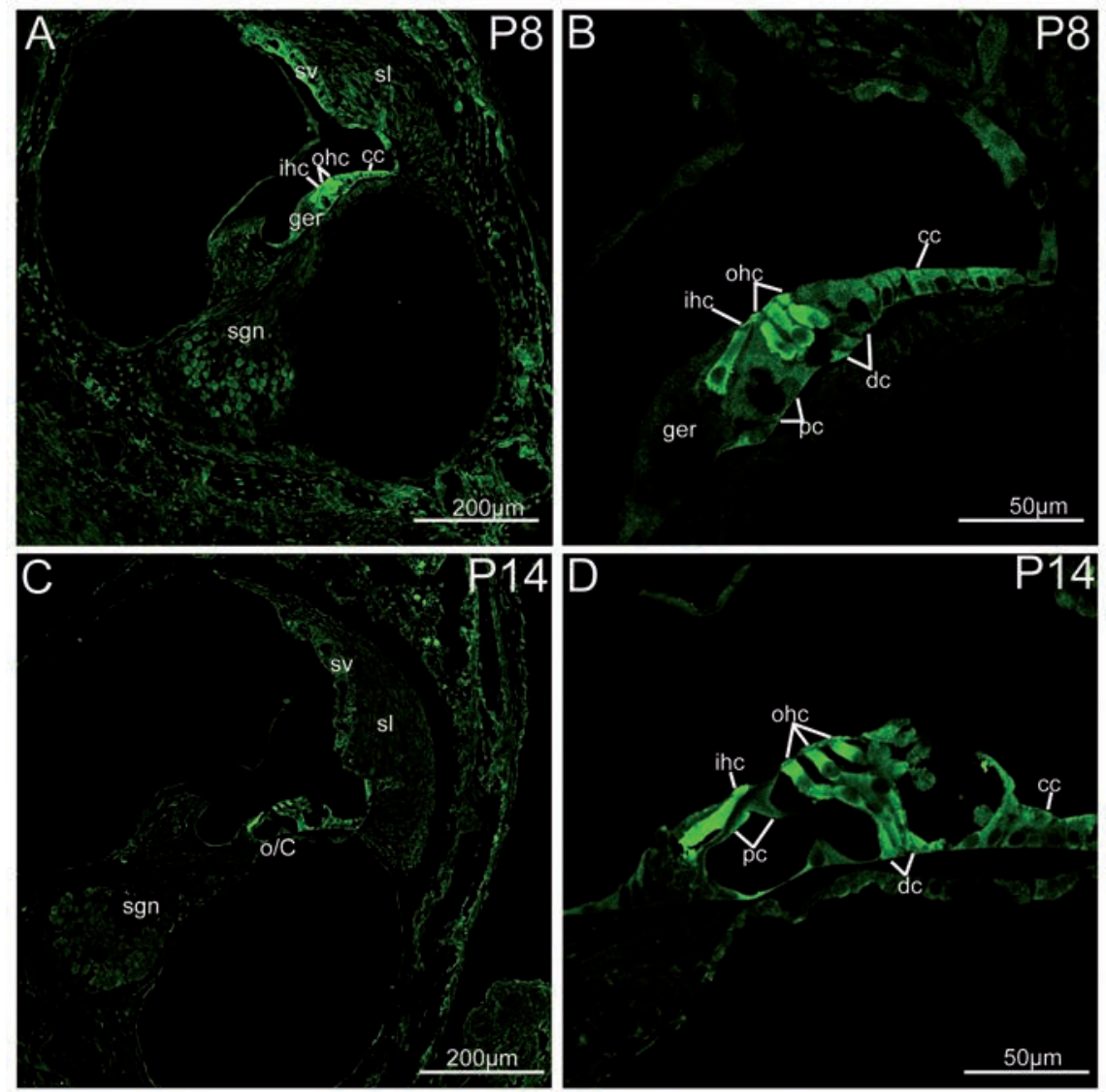

Figure 4. Confocal images of IP3R immunolabeling in the mid turn of the rat cochlea at P8 and P14. A) An overview of IP3R immunolabeling in the mid turn of the rat cochlea at P8; note the pronounced IP3R immunolabeling of the stria vascularis; weak immunolabeling was found in the spiral ganglion neurons. B) Detail of IP3R expression in the P8 organ of Corti; IP3R was localized in the hair cells and Claudius' cells. C) An overview of IP3R immunolabeling in the mid turn of the rat cochlea at P14; strong IP3R immunolabeling was evident in the organ of Corti; however, IP3R immunolabeling in the stria vascularis and spiral ganglion neurons is close to the background level. D) Detail of IP3R expression in the P14 organ of Corti; in the inner hair cells, IP3R labelling was found throughout the cell body; in the outer hair cells, the cytoplasmic region above the nucleus displayed most labelling, Deiters' and Claudius' cells showed weak immunolabeling. ger, greater epithelial ridge; ihc, inner hair cells; ohc, outer hair cells; pc, pillar cell; cc, Claudius' cell; dc, Deiters' cell; o/C organ of Corti; sgn, spiral ganglion neuron; sl, spiral ligament; sv, stria vascularis. 

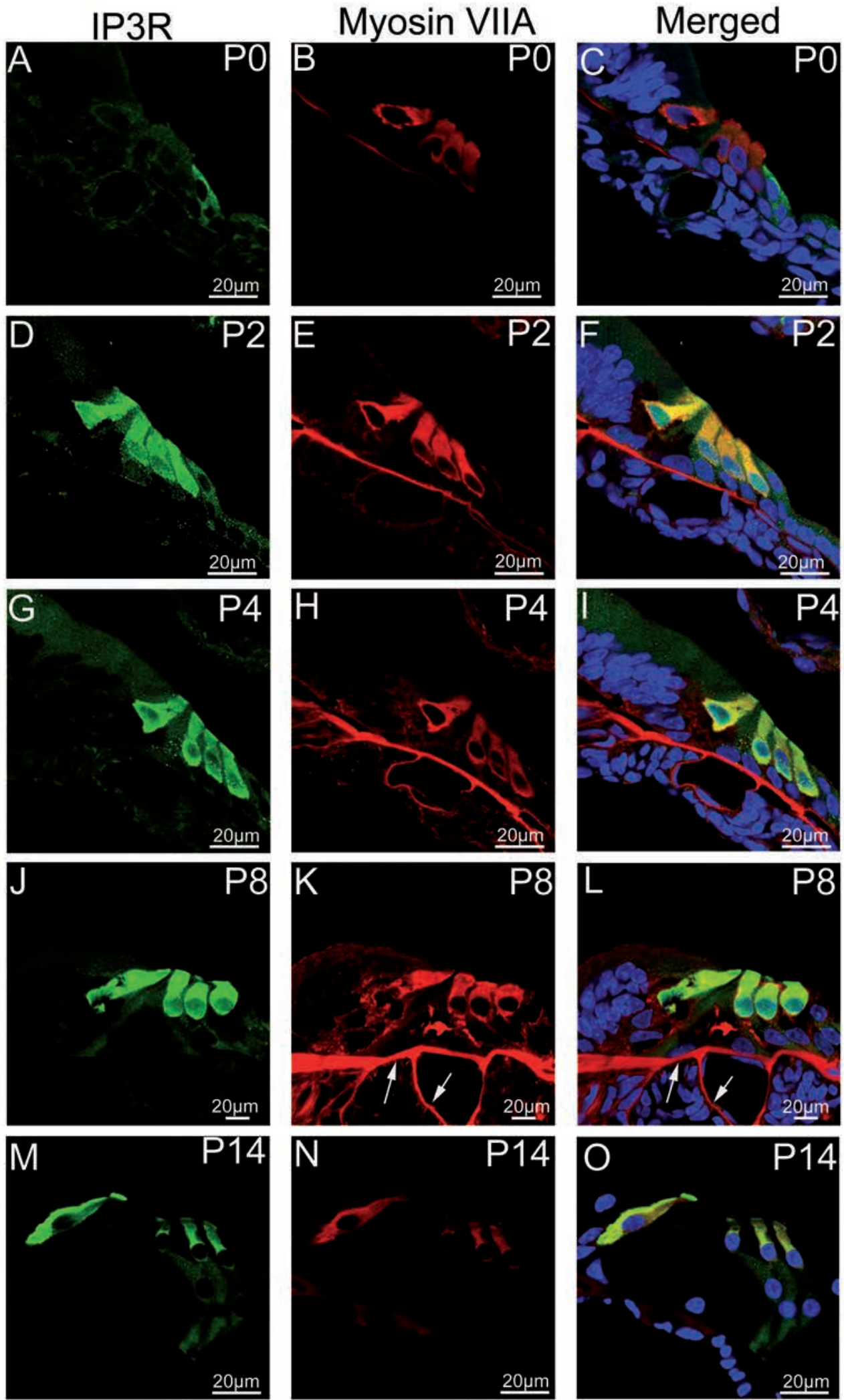

Figure 5. High magnification view of P0, P2, P4, P8 and P14 rat organ of Corti double-labeled by IP3R (green), myosin VIIa (red) under confocal microscopy. A) Immunolabeling for IP3R (green) was only detected in the cytoplasm of the Hensen's cells. B) Inner and outer hair cells were exclusively labelled for myosin VIIa (red). C) No co-expression of IP3R and myosin VIIa could be detected in the merged image, whereas at later stages (P2, P4, P8, P14), double labeling (yellow) was exclusively detected in the hair cell regions (E$\mathrm{O})$. Note that non-specific staining was observed in the basilar membrane and the spiral vessel underneath the basilar membrane (arrows), particularly in the P8 organ of Corti. 
cell of the organ of Corti at birth. It has been reported that at a similar developmental age, delivery of IP3 to a single Hensen's cell in the organotypic cultures of the organ of Corti induced a transient intracellular $\mathrm{Ca}^{2+}$ elevation. Not until P2 was IP3R immunoreactivity detected in both types of hair cells, and persisted into adulthood. The role of IP3R-mediated $\mathrm{Ca}^{2+}$ store release in immature $\mathrm{OHCs}$ is unclear, however, the $\mathrm{Ca}^{2+}$ channel has been demonstrated to trigger the transmitter release at the presynaptic level in IHCs in neonatal mice. ${ }^{21}$ Furthermore, calciuminduced calcium release (CICR) was proposed to contribute to the presynaptic function of neonatal IHCs. ${ }^{22}$ CICR was an amplification process whereby the increase of intracellular calcium caused by the opening of calcium-permeable channels activates calcium release from the stores..$^{23}$ Although CICR depends on RyR, the process of CICR may apply for IP3R as well, given that IP3 is not the only mediator of IP3R function, and that $\mathrm{Ca}^{2+}$ plays a key role in shaping IP3R-evoked $\mathrm{Ca}^{2+}$ signaling. ${ }^{24,25}$ Furthermore, in contrast to localization of RyR at P10 of rat we previously observed, IP3R expression was detectable in IHCs and OHCs 7 days earlier. We proposed that the presence of IP3R in the immature sensory hair cells provided an important pathway to increase intracellular $\mathrm{Ca}^{2+}$ required for functioning and maturation of IHCs and OHCs. At P8, IP3R expression was detected in the supporting cells (predominantly Claudius' cells) in the organ of Corti. The developmental change of IP3R expression in this region observed in present study accurately parallels that of propagation of an IP3-mediated $\mathrm{Ca}^{2+}$ signal across supporting cells of neonatal cochlear organotypic cultures reported previously, namely, the spreading of $\mathrm{Ca}^{2+}$ waves triggered by direct IP3 injection was not observed until P8. ${ }^{26}$ This $\mathrm{Ca}^{2+}$ wave propagation could be eliminated by the gap junction hemichannel blocker. Hemichannel-mediated IP3 release in the developing cochlea has been determined. ${ }^{8}$ On the other hand, in the immature organ of Corti, around P5, activation of IP3-mediated $\mathrm{Ca}^{2+}$ responses could increase the levels of connexin 26 transcripts. ${ }^{27}$ Several studies have proposed that gap junctional communication is required for cochlear ontogeny. ${ }^{28,29}$ Based on these findings, we postulated that IP3 releases through gap junctional hemichannel into the extracellular space, where IP3 acting as an extracellular mediator binds to IP3R on IHCs and OHCs described here to induce $\mathrm{Ca}^{2+}$ release from an IP3-sensitive $\mathrm{Ca}^{2+}$ store, and ultimately increases in intracellular $\mathrm{Ca}^{2+}$ needed for cochlear development. ${ }^{30}$ By extension, impairment of the transference of IP3 through the mutant channels during cochlear ontogeny might be implicated in the etiology of connex-
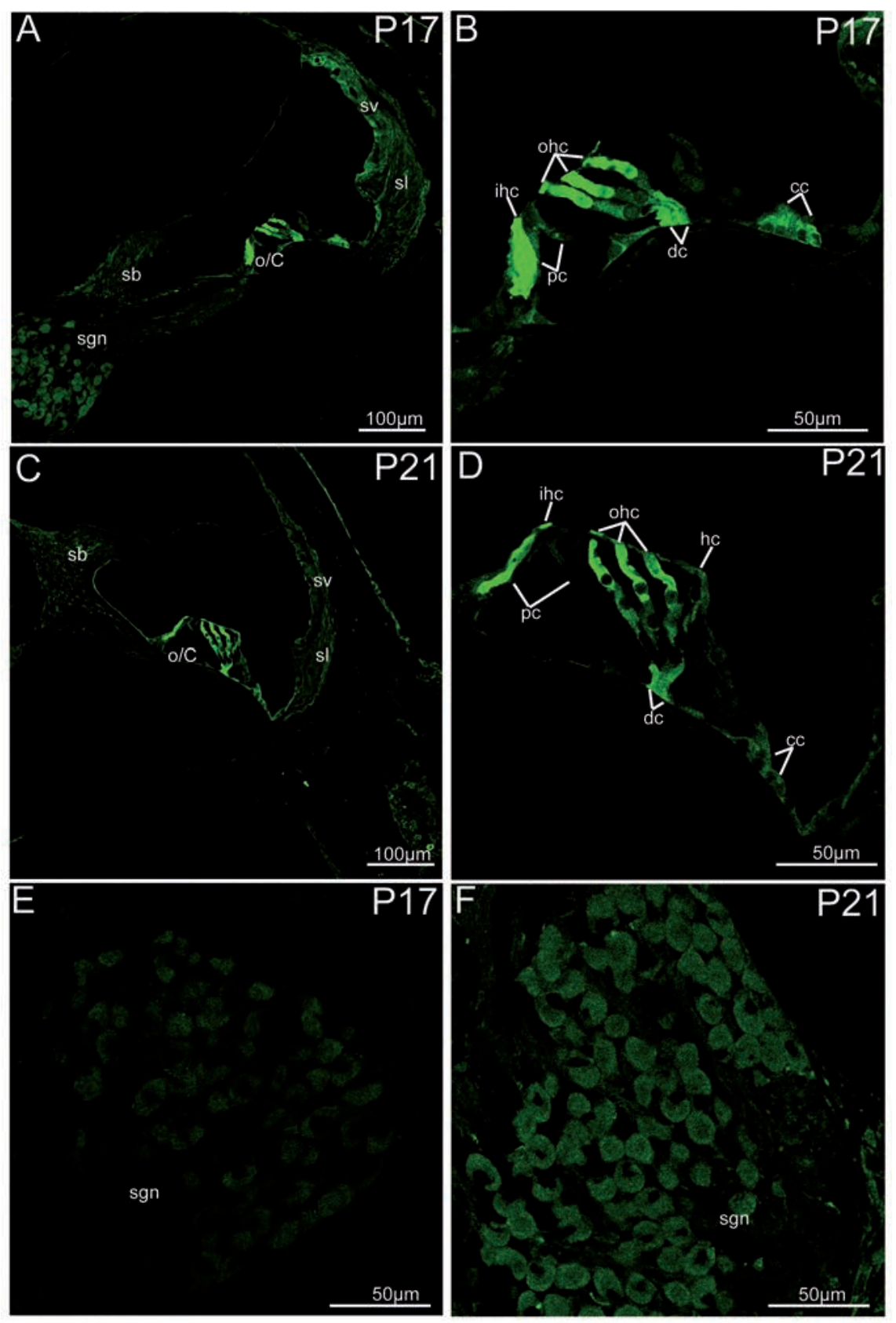

Figure 6. Confocal images of IP3R immunolabeling in the mid turn of the rat cochlea at P17 and P21. A) An overview of IP3R immunolabeling in the mid turn of the rat cochlea at P17; the stria vascularis showed no IP3R immunolabeling; IP3R immunolabeling was constrained to the organ of Corti. B) Detail of IP3R expression in the P17 organ of Corti; strong immunolabeling for IP3R was observed in the inner and outer hair cells; particularly in the inner hair cell, IP3 staining was distributed throughout the whole cytoplasm, from the base to the apex; also in the outer hair cell, IP3R showed a homogenous distribution in the cytoplasm; in addition, the immunolabeling was visible in the basal region of the Deiters' cells adjacent to the basilar membrane, and Claudius' cells. C) An overview of IP3R immunolabeling in the mid turn of the rat cochlea at P21; the organ of Corti maintained strong expression of IP3R. IP3R immunolabeling was absent in the lateral wall of the cochlea. D) Detail of IP3R expression in the P21 organ of Corti; strong IP3R immunolabeling was detected in the cytoplasmic and perinuclear region of the outer hair cell; IP3R continued to express in the cytoplasm of the inner hair cells; in the Deiters' cells, the cytoplasmic region beneath the nucleus showed IP3R immunolabeling; weak IP3R expression was detected in the Claudius' cells in the organ of Corti. E,F) Detail of IP3R expression in the spiral ganglion neurons of P17 and P21 cochlea; IP3R expression in spiral ganglion neurons was marginally above the background level. ihc, inner hair cells; ohc, outer hair cells; cc, Claudius' cell; dc, Deiters' cells; o/C, organ of Corti; sgn, spiral ganglion neuron; sl, spiral ligament; sv, stria vascularis. 
in-related hearing impairment.

After hearing onset, hair cells and supporting cells in the mature cochlea continued to express IP3R, the presence of functional IP3R in these cells has been reported..$^{9,31,32}$ However, inconsistent with our results, there was functional and immunocytochemical evidence for IP3R expression in Hensen's cell of adult guinea-pig and rat cochlea. ${ }^{33}$ The reasons for this discrepancy might include sample preparation, different antibodies used, and some species differences. In the present study, immunofluorescence labeling for IP3R was distributed throughout the cell body of adult IHC, from the base to the apex, indicating the possible involvement of IP3-gated $\mathrm{Ca}^{2+}$ stores in sound transduction at the apex of IHC and in release of neurotransmitter at the base. It was noteworthy that IP3R immunostaining selectively confined to the cytoplasm. Accordingly to our immunofluorescent data, ultrastructural examination of IHCs, OHCs and Deiters' cell has demonstrated that endoplasmic reticulum, the major source for calcium release from intracellular stores, was located in the cytoplasmic region. ${ }^{31,32,34-36}$

Compared with the pronounced expression of IP3R by the sensory hair cells, SGNs expression was relatively weaker. This labeling indicated that IP3-sensitive $\mathrm{Ca}^{2+}$ store may not be a preponderant pathway for $\mathrm{Ca}^{2+}$ entry associated with auditory neurotransmission in the SGNs. In isolated SGNs from the rat cochlea, stimulation of P2Y metabotropic receptors by ATP resulted in the activation of a nonspecific cation conductance. ${ }^{37}$ These receptors are coupled to PLC pathways and, consequently, to IP3 and diacylglycerol (DAG) synthesis. DAG,
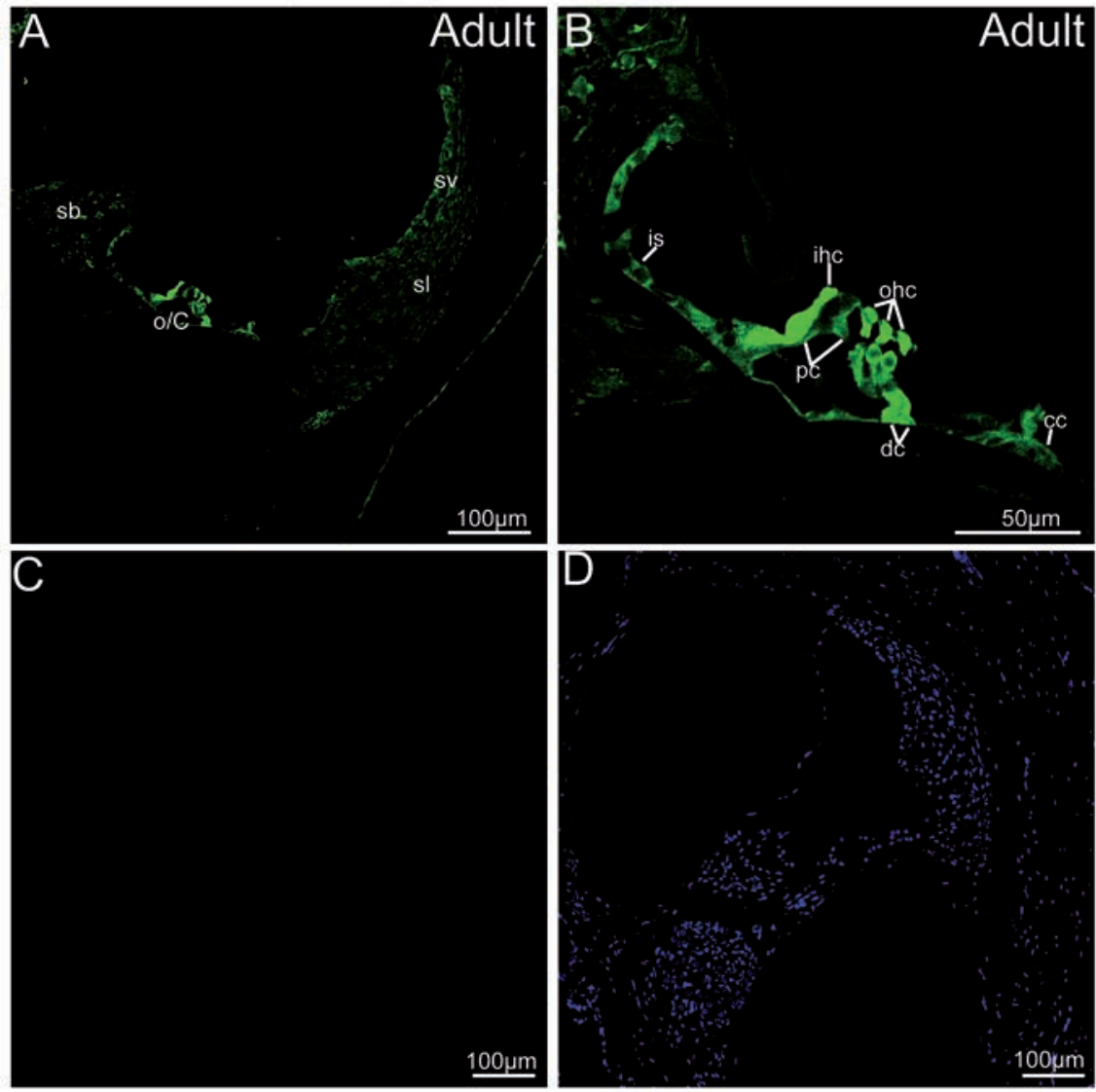

Figure 7. Confocal images of IP3R immunolabeling in the adult rat cochlea and the negative control. A) An overview of IP3R immunolabeling in the mid turn of the rat cochlea; the most prominent IP3R expression was maintained in the organ of Corti; lack of IP3R immunolabeling in the lateral wall of the cochlea. B) Detail of IP3R expression in the adult organ of Corti; in the inner hair cells, IP3R staining was distributed throughout the whole cell; in the outer hair cells, IP3R immunoreactivity was localized more uniformly within the cytoplasm; note that the apical cytoplasm of the outer hair cell was also strongly immunolabeled; IP3R immunolabeling was evident at a high level in the Deiters' cells; furthermore, the inner sulcus cells surrounding the inner hair cells and Claudius' cells were also labeled. C,D) The absence of IP3R immunofluorescence in negative control. ihc, inner hair cells; ohc, outer hair cells; cc, Claudius' cell; dc, Deiters' cells; o/C, organ of Corti; is, inner sulcus cell; sl, spiral ligament; sv, stria vascularis.

another important intracellular messenger leading to cation channel opening, is formed concurrently with IP3 during PLC activation. ${ }^{38}$ It has been reported that DAG-sensitive members of the transient receptor potential (TRP) channel family were involved in the $\mathrm{G}$ proteincoupled receptor signaling in the SGNs. ${ }^{39,40}$ TRP receptors have been shown to be strongly expressed in the cell body of SGNs by immunohistochemistry as well..$^{41}$ In addition, the most pronounced RyR protein expression, involving all three isoforms, occurred in the cell bodies of the SGNs throughout all post-natal developmental stage. Moreover, electrophysiological findings showed RyR-gated $\mathrm{Ca}^{2+}$ release evoked an increase in intracellular $\mathrm{Ca}^{2+}$ concentration in the cell body of the SGNs. ${ }^{42}$ These differences between IP3R and RyR might indicate that they are involved in distinct physiological events.

The present results revealed for the first time by RT-PCR the presence of mRNA for all the three isoforms of IP3R, in rat cochlear tissues, at four developmental stages. In contrast to the differential expression of IP3R protein in developing rat cochlea, no temporal variation in the expression of mRNA for the three isoforms of IP3R was detectable. The results

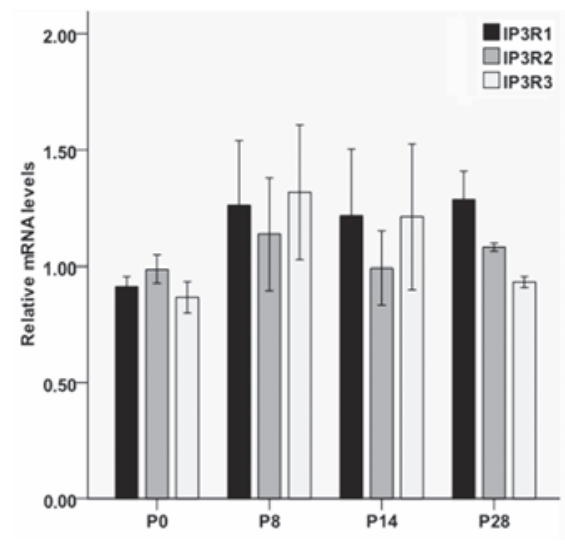

Figure 8. RT-PCR detection of mRNA for three IP3R isoforms, IP3R1, IP3R2, and IP3R3, at four different developmental stages of the rat cochlea, including P0, P8, P14, and P28. All three isoforms of IP3Rs mRNAs were detected at all developmental stages examined, using samples from whole cochlear tissues. There were no differences in expression of IP3R1, IP3R2 and IP3R3 mRNA at the various developmental stages $(P I P 3 R 1=0.589, P I P 3 R 2=$ 0.865 , and PIP3R3=0.442), and there was no significant difference among IP3R1, IP3R2, and IP3R3 mRNA levels at each corresponding stage $(\mathrm{PPO}=0.392, \mathrm{PP} 8=$ 0.893 , PP14=0.795, and PP28=0.127). 
are compatible with the observation that the relative mRNA levels for each IP3R isoform, evaluated by ratiometric PCR methods, did not directly correlate with protein levels..$^{43}$ At each corresponding developmental stage, no statistically significant difference was found among all the three IP3R isoforms. It is unclear whether it implies that there is no difference in the subcellular localization of these IP3R subtypes. Further studies are clearly merited to resolve this issue.

In this study, we determined the spatiotemporal expression of IP3R in the developing rat cochlea. IP3R expression occurred in the very early post-natal period, at the protein and mRNA levels, and was maintained throughout the period of hearing acquisition, implying a role for IP3R in cochlear development and auditory function. The expression of IP3R in the sensory hair cells and supporting cells of the developing organ of Corti further supported the involvement of IP3-gated intracellular $\mathrm{Ca}^{2+}$ stores in sound transduction and the regulation of cochlear micromechanics.

\section{References}

1. Rodriguez L, Simeonato E, Scimemi P, Anselmi F, Cali B, Crispino G, et al. Reduced phosphatidylinositol 4,5-bisphosphate synthesis impairs inner ear $\mathrm{Ca} 2+$ signaling and high-frequency hearing acquisition. Proc Natl Acad Sci USA 2012; 109:14013-8.

2. Mammano F. ATP-dependent intercellular $\mathrm{Ca} 2+$ signaling in the developing cochlea: facts, fantasies and perspectives. Semin Cell Dev Biol 2013;24:31-9.

3. Berridge MJ. Elementary and global aspects of calcium signalling. J Physiol 1997; 499(Pt 2):291-306.

4. Beltramello M, Piazza V, Bukauskas FF, Pozzan T, Mammano F. Impaired permeability to Ins(1,4,5)P3 in a mutant connexin underlies recessive hereditary deafness. Nat Cell Biol 2005;7:63-9.

5. Ehrlich BE. Functional properties of intracellular calcium-release channels. Curr Opin Neurobiol 1995;5:304-9

6. Foskett JK, White C, Cheung KH, Mak DO. Inositol trisphosphate receptor $\mathrm{Ca} 2+$ release channels. Physiol Rev 2007;87:593-658.

7. Miyakawa T, Maeda A, Yamazawa T, Hirose K, Kurosaki T, lino M. Encoding of Ca2+ signals by differential expression of IP3 receptor subtypes. EMBO J 1999;18:1303-8.

8. Gossman DG, Zhao HB. Hemichannelmediated inositol 1,4,5-trisphosphate (IP3) release in the cochlea: a novel mechanism of IP3 intercellular signaling. Cell Commun Adhes 2008;15:305-15.
9. Sugasawa M, Erostegui C, Blanchet C, Dulon D. ATP activates non-selective cation channels and calcium release in inner hair cells of the guinea-pig cochlea. J Physiol 1996;491(Pt 3):707-18.

10. Li W, Llopis J, Whitney M, Zlokarnik G, Tsien RY. Cell-permeant caged InsP3 ester shows that $\mathrm{Ca} 2+$ spike frequency can optimize gene expression. Nature 1998;392: 936-41.

11. Siefjediers A, Hardt M, Prinz G, Diener M. Characterization of inositol 1,4,5-trisphosphate (IP3) receptor subtypes at rat colonic epithelium. Cell Calcium 2007;41: 303-15.

12. Huang G, Bartlett PJ, Thomas AP, Moreno SN, Docampo R. Acidocalcisomes of Trypanosoma brucei have an inositol 1,4,5trisphosphate receptor that is required for growth and infectivity. Proc Natl Acad Sci USA 2013;110:1887-92.

13. Parrington J, Brind S, De Smedt H, Gangeswaran R, Lai FA, Wojcikiewicz R, et al. Expression of inositol 1,4,5-trisphosphate receptors in mouse oocytes and early embryos: the type I isoform is upregulated in oocytes and downregulated after fertilization. Dev Biol 1998;203:451-61.

14. Liang Y, Huang L, Yang J. Differential expression of ryanodine receptor in the developing rat cochlea. Eur J Histochem 2009;53:249-60

15. Liu WJ, Yang J. Preferentially regulated expression of connexin 43 in the developing spiral ganglion neurons and afferent terminals in post-natal rat cochlea. Eur J Histochem 2015;59:2464.

16. Simonneau L, Gallego M, Pujol R. Comparative expression patterns of T-, $\mathrm{N}-$, E-cadherins, beta-catenin, and polysialic acid neural cell adhesion molecule in rat cochlea during development: implications for the nature of Kolliker's organ. J Comp Neurol 2003;459:113-26.

17. El-Amraoui A, Cohen-Salmon M, Petit C, Simmler MC. Spatiotemporal expression of otogelin in the developing and adult mouse inner ear. Hear Res 2001;158:151-9.

18. Bermingham-McDonogh 0, Oesterle EC, Stone JS, Hume CR, Huynh HM, Hayashi T. Expression of Proxl during mouse cochlear development. J Comp Neurol 2006;496:172-86.

19. Hasson T, Gillespie PG, Garcia JA, MacDonald $\mathrm{RB}$, Zhao Y, Yee AG, et al. Unconventional myosins in inner-ear sensory epithelia. J Cell Biol 1997;137:1287-307.

20. Geal-Dor M, Freeman S, Li G, Sohmer H. Development of hearing in neonatal rats: air and bone conducted ABR thresholds. Hear Res 1993;69:236-42.

21. Beutner D, Moser T. The presynaptic function of mouse cochlear inner hair cells during development of hearing. J Neurosci 2001;21:4593-9.

22. Kennedy HJ, Meech RW. Fast Ca2+ signals at mouse inner hair cell synapse: a role for $\mathrm{Ca} 2+$-induced $\mathrm{Ca} 2+$ release. J Physiol 2002;539:15-23.

23. Williams AJ. Ion conduction and selectivity in the ryanodine receptor channel. Front Biosci 2002;7:d1223-30.

24. Missiaen L, Taylor CW, Berridge MJ. Spontaneous calcium release from inositol trisphosphate-sensitive calcium stores. Nature 1991;352:241-4.

25. Berridge MJ. Inositol trisphosphate and calcium signalling. Nature 1993;361:315-25.

26. Zhang Y, Tang W, Ahmad S, Sipp JA, Chen $\mathrm{P}$, Lin X. Gap junction-mediated intercellular biochemical coupling in cochlear supporting cells is required for normal cochlear functions. Proc Natl Acad Sci USA 2005;102:15201-6.

27. Ortolano S, Di Pasquale G, Crispino G, Anselmi F, Mammano F, Chiorini JA. Coordinated control of connexin 26 and connexin 30 at the regulatory and functional level in the inner ear. Proc Natl Acad Sci USA 2008;105:18776-81.

28. Chen J, Chen J, Zhu Y, Liang C, Zhao HB. Deafness induced by Connexin 26 (GJB2) deficiency is not determined by endocochlear potential (EP) reduction but is associated with cochlear developmental disorders. Biochem Biophys Res Commun 2014;448:28-32.

29. Kelly JJ, Forge A, Jagger DJ. Development of gap junctional intercellular communication within the lateral wall of the rat cochlea. Neuroscience 2011;180:360-9.

30. Engel J, Michna M, Platzer J, Striessnig J. Calcium channels in mouse hair cells: function, properties and pharmacology. Adv Otorhinolaryngol 2002;59:35-41.

31. Lagostena L, Mammano F. Intracellular calcium dynamics and membrane conductance changes evoked by Deiters' cell purinoceptor activation in the organ of Corti. Cell Calcium 2001;29:191-8.

32. Mammano F, Frolenkov GI, Lagostena L, Belyantseva IA, Kurc M, Dodane V, et al. ATP-Induced $\mathrm{Ca}(2+)$ release in cochlear outer hair cells: localization of an inositol triphosphate-gated $\mathrm{Ca}(2+)$ store to the base of the sensory hair bundle. $\mathrm{J}$ Neurosci 1999;19:6918-29.

33. Lagostena L, Ashmore JF, Kachar B, Mammano F. Purinergic control of intercellular communication between Hensen's cells of the guinea-pig cochlea. J Physiol 2001;531:693-706

34. Spicer SS, Thomopoulos GN, Schulte BA. Novel membranous structures in apical and basal compartments of inner hair cells. J Comp Neurol 1999;409:424-37. 
35. Solovyova N, Verkhratsky A. Neuronal endoplasmic reticulum acts as a single functional $\mathrm{Ca} 2+$ store shared by ryanodine and inositol-1,4,5-trisphosphate receptors as revealed by intra-ER $[\mathrm{Ca} 2+]$ recordings in single rat sensory neurones. Pflugers Arch 2003;446:447-54.

36. Ross CA, Meldolesi J, Milner TA, Satoh T, Supattapone S, Snyder SH. Inositol 1,4,5trisphosphate receptor localized to endoplasmic reticulum in cerebellar Purkinje neurons. Nature 1989;339:468-70.

37. Ito K, Dulon D. Nonselective cation conductance activated by muscarinic and purinergic receptors in rat spiral ganglion neurons. Am J Physiol Cell Physiol 2002;282:C1121-35.

38. Moran MM, Xu H, Clapham DE. TRP ion channels in the nervous system. Curr Opin Neurobiol 2004;14:362-9.

39. Bauer CA, Brozoski TJ, Myers KS. Acoustic injury and TRPV1 expression in the cochlear spiral ganglion. Int Tinnitus $\mathrm{J}$ 2007;13:21-8.

40. Ishibashi T, Takumida M, Akagi N, Hirakawa K, Anniko M. Changes in transient receptor potential vanilloid (TRPV) 1, 2, 3 and 4 expression in mouse inner ear following gentamicin challenge. Acta Otolaryngol 2009;129:116-26.

41. Phan PA, Tadros SF, Kim Y, Birnbaumer L, Housley GD. Developmental regulation of TRPC3 ion channel expression in the mouse cochlea. Histochem Cell Biol 2010;133:437-48.

42. Morton-Jones RT, Cannell MB, Jeyakumar LH, Fleischer S, Housley GD. Differential expression of ryanodine receptors in the rat cochlea. Neuroscience 2006;137:275-86.

43. Taylor CW, Genazzani AA, Morris SA. Expression of inositol trisphosphate receptors. Cell Calcium 1999;26:237-51. 